\title{
Space Charge Measurements in Thin Bilayer BOPP Films by Thermal Pulse Method
}

\author{
Feihu Zheng, Jiao Xie, Zhenlian An and Yewen Zhang \\ Tongji University \\ Department of Electrical Engineering \\ Shanghai, 201804, China \\ Gilbert Teyssèdre \\ University Paul Sabatier Toulouse and CNRS \\ LAPLACE (Laboratoire Plasma et Conversion d'Énergie) \\ 118 route de Narbonne, F-31062 Toulouse cedex 9, France
}

\begin{abstract}
In this paper, space charge accumulation in bilayer dielectrics composed of two metalized biaxially oriented polypropylene (BOPP) films thermally coupled by thin heat transfer oil (dibenzyltoluene, DBT) is investigated by thermal pulse method. An improved thermal pulse method for in-situ double-sided measurements is proposed to achieve higher spatial resolution. The accumulation and decay of the injected space charge is monitored after the laminated films being subjected to DC applied field of $150 \mathrm{~V} / \mu \mathrm{m}$ at various temperatures from $25{ }^{\circ} \mathrm{C}$ to $70{ }^{\circ} \mathrm{C}$. Experimental results show that both charge injection rate and migration are sensitive to the operated temperature. Bipolar space charge injection is the rule, but negative charge becomes dominant as the injection rate asymmetry enhances with the elevated temperature. The interface between the films acts as a potential barrier to space charge transport at relative low temperature and the effect of the potential barrier appears weaken as the temperature rises. Therefore, the maximum electrical field by accumulated space charge is approximately $80 \mathrm{~V} / \mu \mathrm{m}$ and the internal interface has little impact at $70^{\circ} \mathrm{C}$.
\end{abstract}

Index Terms - thermal pulse method, space charge, dielectric interface, BOPP, capacitors

\section{INTRODUCTION}

INSULATING polymer films are widely used as capacitor dielectrics. Nowadays, bilayer or multi-layer composite dielectric films have attracted research interests, because they may overcome the shortcomings of single-layered films or gain the performances that single-layered films cannot achieve [1]. Compared with film capacitors with single-layer dielectrics, film capacitors with bilayer or multi-layer dielectrics have important advantages, such as promoted withstanding temperature and withstanding field, enhanced energy storage density, improved mechanical strength, higher-precision capacity, lighter weight and smaller size [2]. Panasonic Corporation developed film capacitor with extremely small volume and extremely high capacity by depositing polyphenylene oxide (PPO) thin layer (less than $0.5 \mu \mathrm{m}$ ) on a double-sided metalized polyphenylene sulfide (PPS) film [3]. This example demonstrates the advantage of bilayer film as capacitor dielectrics.
It is well known that there could be a certain amount of space charge accumulation for bilayer or single-layer insulation being suffered to DC high field. This would result in the distortion of internal electric field, and then may affect insulation performance of films. Especially for double-layer dielectrics, the interface is frequently considered as a weakness for the insulation. For example, the accessories such as joints and terminals in cable systems form a composite (bilayer) insulation structure, which is thought to be the weakest link in the entire cable insulation system due to the existence of interfaces [4]. Compared with the bulk insulation, the interfaces often play a vital role in the dynamic evolution of space charge due to specific electrical properties. From a microscale perspective, molecular conformation disorders, oxidation or impurity decomposition and so on will form new trap levels at the interface making it different from the bulk [5]. From a macro perspective, flatness of the surfaces, contact pressure or coupling modes between the interfaces, will affect the interface behavior [6]. The Maxwell-Wagner (MW) effect [7-9] accounts for space charge accumulation at the discontinuous interface of two different materials. Only when the materials are identical there should be no space charge accumulation at the interface. However, several studies have found charge accumulation at the interface between two identical materials, or charge in an amount 
not expected by MW effect. It is thought to be due to the discontinuous distribution of trap sites arising from different degrees of micro or macro defects of chemical or physical origins $[10,11]$.

Many groups have carried out research on the space charge and interface characterization of double-layered dielectrics. T. Tanaka et al [12] investigated the interface behavior of double-layered dielectrics composed of low-density polyethylene (LDPE) and ethylene vinyl-acetate (EVA) as early as 1996 based on the laser induced pressure wave propagation (LIPWP) method. They found that the accumulation and migration of charge at the interface is related to space charge formation in each singlelayered material. Bodega et al [13] studied charge behavior at the coaxial interface of cross-linked polyethylene (XLPE) / ethylene-propylene rubber (EPR) under temperature gradient by the pulsed electro-acoustic method (PEA) method. They found that the build-up of space charge at the interface is strongly related to the temperature in the both materials. Study by Yin et al [14] have shown that the charge at the interface of LDPE / EPR laminates consists of the polarized charge by the MW effect and the injected charge from the cathode. Vu et al obtained interface charge amount, sign and build-up or decay kinetics consistent with MW effect in XLPE/EPR bilayers [15, 16].

Rogti et al [5] investigated bi-layers of XLPE/XLPE films, and found that the interface charge does not conform to the Maxwell-Wagner effect; all the charges at the internal interface come from the electrode injection. Das et al [17] carried out research on the interface charge of double-layered dielectrics of polyethylene and epoxy resin under designed interface conditions, and showed that the charge amount at the interface depends on the surface state of the two materials. The air layer between the interfaces has a substantial impact on the charge evolution and charge polarity at the interface.

However, the results mentioned above mainly concern the interface characterization of the composite dielectrics of cable systems or thick sheets and the total thickness of the sample always exceeds several hundred of microns. There are few studies on the space charge and interface characterization of bilayer dielectric thin films with a total thickness of less than $20 \mu \mathrm{m}$. In the current investigation, space charge behavior in bilayer biaxially oriented polypropylene (BOPP) films with total thickness of $13.6 \mu \mathrm{m}$ is measured by the thermal pulse method. The impact of experimental temperature on the space charge and interface behavior of bilayer dielectrics under applied high electric fields is discussed.

\section{EXPERIMENTAL}

\subsection{SAMPLE PREPARATION}

Commercially available BOPP films for capacitor with thickness 9.8 and $3.8 \mu \mathrm{m}$ are used in this study. Both kinds of films are with the size of $5 \times 5 \mathrm{~cm}^{2}$ from the same company. The $9.8 \mu \mathrm{m}$ film and the $3.8 \mu \mathrm{m}$ film are with coarsened surface on one side and double sides, respectively. Surface coarsening treatment is achieved to improve oil impregnation.

Two kinds of bilayer samples are prepared as shown in Figure 1. For sample A, the two films are aluminum (Al) metalized on both faces with a diameter of $5 \mathrm{~mm}$ and thickness $180 \mathrm{~nm}$, and then the films are thermally coupled from rough surface sides by a thickness controllable very thin layer of dibenzyltoluene (DBT) oil to minimize the impact of heat loss at the interface during thermal pulse measurements. The thickness of the DBT layer is far less than $0.5 \mu \mathrm{m}$. For sample $\mathrm{B}$, the $9.8 \mu \mathrm{m}$ film is Al- metalized with a diameter of $5 \mathrm{~mm}$ on the flat side. Al was evaporated on the rough face of the $3.8 \mu \mathrm{m}$ thick film. The free sides of the two films are also thermally coupled by DBT. The prepared bilayer samples are pressed with flat and heavy steel plate to further reduce the DBT thickness prior to the measurements.

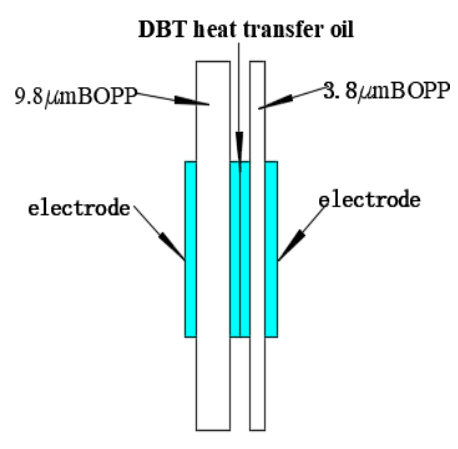

(a)

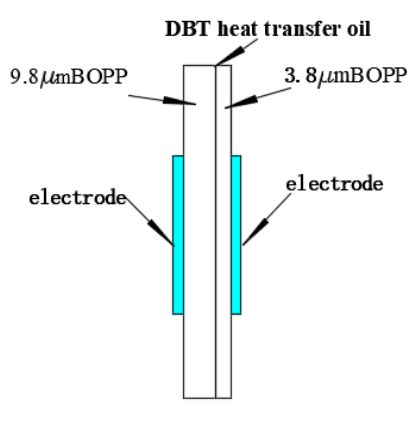

(b)
Figure 1. Schematic diagram of bilayer samples: (a) sample A, (b) sample B.

\subsection{EXPERIMENTAL PRINCIPLE AND SETUP}

The thermal pulse method is a powerful tool to detect space charge profiles within thin dielectric films. The measurement principle [18] is as follows: the laser light pulse shoots the metalized electrode, and then the electrode generates heat pulses and transmits it to the sample after partially absorbing laser pulses. The displacement of space charge in the dielectric film due to the local non-uniform deformation during the heat pulse transmission across the sample induces a displacement current in the external circuit. Electric field distribution and charge density distribution can be finally retrieved from the current signal through the deconvolution algorithm.

Several techniques such as approximation method [19], Tikhonov regularization method [20, 21], Monte Carlo method[22] have been used to analysis the thermal pulse data. No matter which algorithm is used, the obtained spatial resolution is greatly determined by the local temperature gradient caused by thermal pulse disturbance. However, there is inevitable pulse dispersion as the thermal pulse transmits over deeper distance, which leads to the decrease of temperature gradient, and subsequently the reduction of the spatial resolution.

An effective way to compensate the loss of the resolution due to the decreasing temperature gradient along the total thickness is to perform the measurement from both sides of the sample. Subsequently, the retrieved space charge or electric field data from each side can be combined at the middle depth to obtain higher spatial resolution. However, the combination of the data at the middle depth may bring about the non-continuity of the curves, even if the measurements are achieved nearly simultaneously during a same stressing step. Many factors on the sides of the sample such as the stability of the pulse, the 
absorptivity of incident light by the target electrode, surface heat loss of target electrode would lead to the difference of the temperature gradient at the same location and therefore to the difference in thermal pulse-induced current. The features of non-continuity in the field distribution can be found in the figures in the section 3 . The schematic diagram of thermal pulse measurements system is shown in Figure 2. To achieve the insitu double-sided measurements, the incident pulse laser is divided by $5 / 5$ splitter. It is deviated to successively shot onto the metalized electrodes. Extra parts composed by a DC power source, a protective resistance $\mathrm{R}$ and a blocking capacitor $\mathrm{C}$, are supplemented into the traditional measuring circuit. The switch and the protecting circuit reduce the breakdown risk of the current pre-amplifier. The bandwidth of the current preamplifier is from DC to $300 \mathrm{kHz}$.

This improved experimental setup makes full use of the feature that the resolution of the TP method near the heated surface of the dielectric film is the highest: the double-sided online measurement without inverting the sample under applied voltages is realized. The pulse width of the laser is $7 \mathrm{~ns}$ with single pulse energy $1 \mathrm{~mJ}$. A small part of laser pulse divided by $1 / 9$ splitter is collected on a paper-covered photodiode to generate the trigger signal. The main part of the laser is split into two beams. The double-layered sample is clamped by a pair of brass ring electrodes, and is kept flat while being installed.

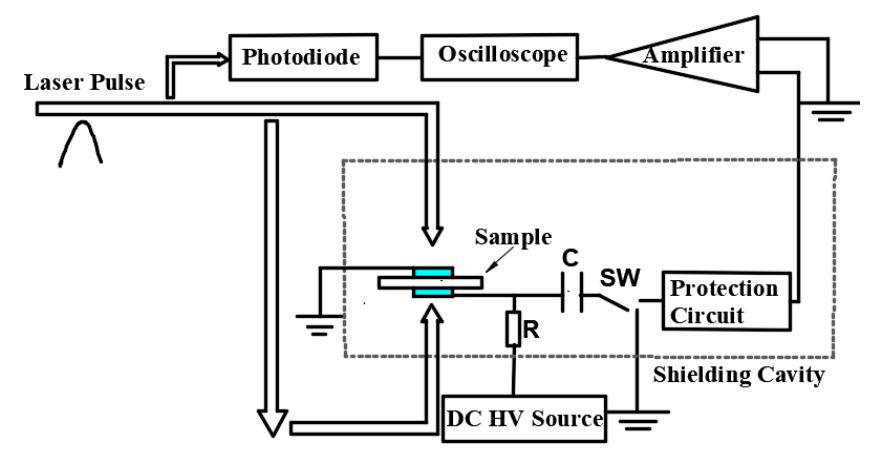

Figure 2. Schematic diagram of the thermal pulse measurements system.

\subsection{MEASUREMENT PROTOCOL}

For sample A, the measurements with voltage-on and voltageoff were performed at $25^{\circ} \mathrm{C}, 40^{\circ} \mathrm{C}$ and $70^{\circ} \mathrm{C}$. For sample B, the measurements with voltage-on and voltage-off were performed only at $25^{\circ} \mathrm{C}$. The specific measuring protocol at each temperature is shown in Figure 3. An electric field of $10 \mathrm{~V} / \mu \mathrm{m}$ was used as the calibration field for determining instrument and material parameters. The electric fields were step wisely increased from $10 \mathrm{~V} / \mu \mathrm{m}$ to $150 \mathrm{~V} / \mu \mathrm{m}$, and applied for about 3 min at each step to confirm that the tested sample can withstand such an electric stress. The samples were then subjected to the electric field of $150 \mathrm{~V} / \mu \mathrm{m}$ for 2 hours. Finally the charge in the sample was also monitored for 2 hours after the removal of electric field of $150 \mathrm{~V} / \mu \mathrm{m}$. The electric field intensity is defined as negative on the axis when the polarity of the applied voltage is positive (see Figure 2). In this study, the temperature of the sample cavity was controlled by means of infrared radiant lamp heating during the whole measurements, and the test did not start until the thermal equilibrium was

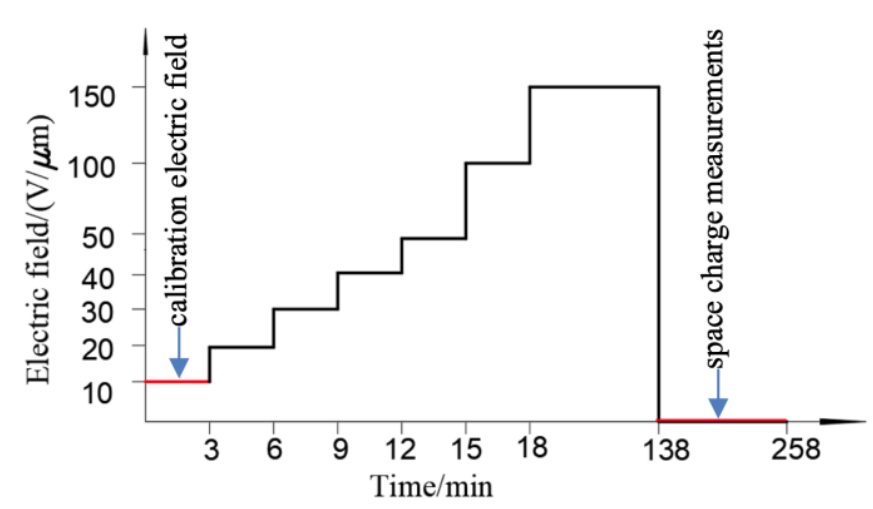

Figure 3. the protocol for space charge measurements.

reached.

\section{RESULTS}

This section mainly discusses the effect of the ambient temperature on the space charge and the interface behavior of sample A from the view of electric field profiles. The outer surface of the $9.8 \mu \mathrm{m}$-thick BOPP film of the bilayer sample A is connected to the ground electrode as the cathode, and the outer surface of the $3.8 \mu \mathrm{m}$-thick BOPP film is linked to the high voltage electrode as the anode. The current signals of the sample as response to thermal excitation are deconvoluted to obtain electric field distributions based on the scale transformation method (ST), which is also known as the approximation method. Considering the oscillation after derivation of the field, only the field distribution is shown.

\subsection{FIELD DISTRIBUTION IN SAMPLE A AT $25^{\circ} \mathrm{C}$}

Figure 4 shows the electric field distribution for bilayer sample A obtained at $25^{\circ} \mathrm{C}$. The double-sided TP measurements are assembled at a depth of about $7 \mu \mathrm{m}$ to build the full profile. As can be seen from Figure 4, a very obvious residual electric field appears in the bulk of the sample immediately after the removal of the $150 \mathrm{~V} / \mu \mathrm{m}$ electric field. The maximum of the residual electric field appears near the cathode in the $9.8 \mu \mathrm{m}$ thick BOPP film, which is close to $30 \mathrm{~V} / \mu \mathrm{m}$. The residual electric field distribution does not change much within the subsequent 2 hours, which indicates that the charge decay is very slow. One can find the slope of the electric field adjacent to the cathode is negative and tends to decrease slightly with time. According to the Poisson's equation, there are lots of negative charges accumulating in the region from the outer surface of $9.8 \mu \mathrm{m}$-thick BOPP film to about $3.5 \mu \mathrm{m}$ depth in sample A. The mean negative charge density is about 200 $\mu \mathrm{C} / \mathrm{cm}^{3}$ in the charge accumulation region. There is no obvious space charge accumulation found in the region between $3.5 \mu \mathrm{m}$ depth and the internal interface. The decrease of the slope of the charge accumulation region indicates that the space charge is driven by the residual field to the cathode.

From the thinner layer side, there is large amount of positive charges accumulating in the region from the internal interface to $11.8 \mu \mathrm{m}$ depth $(\approx 1 \mu \mathrm{m}$ from anode). The mean positive charge density is about $120 \mu \mathrm{C} / \mathrm{cm}^{3}$. No obvious positive charges are found in the region adjacent to the anode. It is 


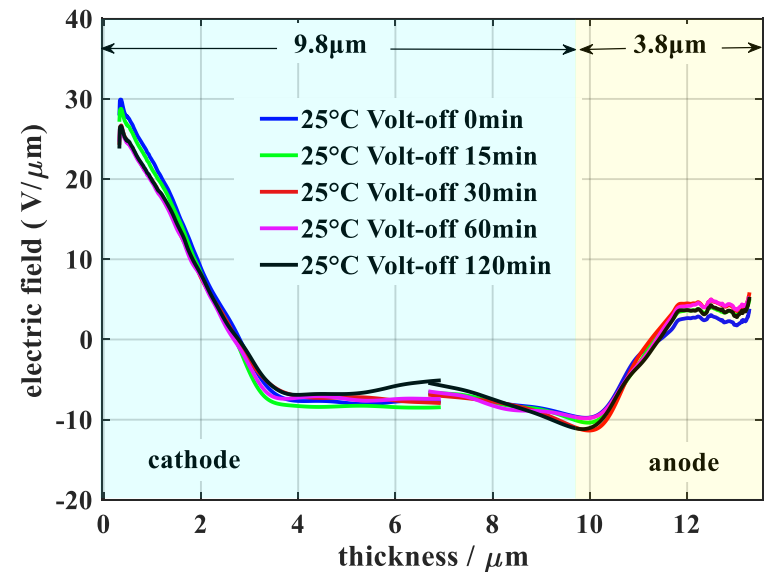

Figure 4. Electric field distribution of sample A during voltage-off at $25^{\circ} \mathrm{C}$.

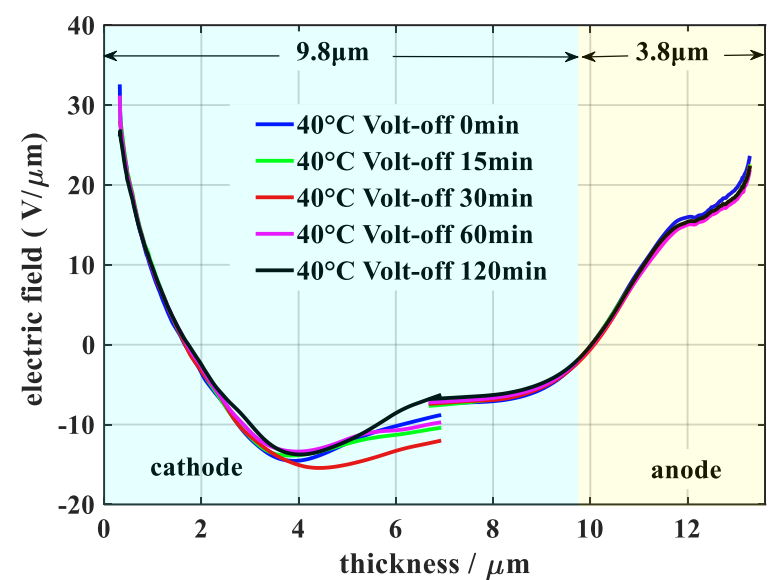

Figure 5. Electric field distribution of sample A during voltage-off at $40{ }^{\circ} \mathrm{C}$. thought that the injected positive charge is driven by the applied field. But the migration of the positive charge with possible relative high mobility, is blocked at the interface. This could be the reason for not observing positive charge adjacent to the anode.

From Figure 4, the amount of negative charge accumulating in the $9.8 \mu \mathrm{m}$-thick BOPP film bulk is clearly greater than that of the positive charge accumulating in the $3.8 \mu \mathrm{m}$-thick BOPP film. This is because the applied electric field brings about the declining deformation of the interface barrier, which makes the potential barrier height for hole injection higher than for electron injection [23]. Therefore, the charge injection rate of positive charge would be weaker as found in Figure 4. This is believed to be the reason for the asymmetric distribution of space charge in this $25^{\circ} \mathrm{C}$ case.

On the contrary, it is interesting to find the apparent almost symmetric extending depth for both polarities injected charges. Charge migration is thought to be modulated by trap level and trap density. The injected negative charge is constantly captured by traps during the migration process, and the modulation of the charge traps limits the migration distance during the 2 hours for the sample being subjected to the applied electric field. Regarding the similar dynamic mechanisms of positive and negative charges, it may be fortuitous. Indeed, there are two possibilities with injected positive charge, being either stopped at the internal interface or having the same velocity as negative injected charges. These possibilities should be unraveled in the following parts.

\subsection{FIELD DISTRIBUTION IN SAMPLE A AT $40^{\circ} \mathrm{C}$}

Figure 5 shows the electric field profiles in sample A immediately after the removal of applied voltage at $40^{\circ} \mathrm{C}$. By comparing the electric field profiles shown in Figure 4 and Figure 5, it is clear that the sample A still shows asymmetrical bi-polar space charge injection. The asymmetrical bi-polar charge injection is also reported in single-layer BOPP film [24]. However, the accumulating charge density and the charge extending depth have changed significantly.

Supposing that the Schottky emission occurs at the cathode and the anode during the voltage on, higher charge injecting rate as the result of the thermal-aided emission is expected with increasing temperature. At the $9.8 \mu$ m-thick BOPP film side, though the maximum of the residual field does not change much compared to $25^{\circ} \mathrm{C}$ data, the steeper slope of the electric field indicates higher negative charge density. In addition, the negative charge accumulation region extends to about $4 \mu \mathrm{m}$ depth. The total negative charge is obviously higher than that in the case of $25^{\circ} \mathrm{C}$, which is consistent with the above analysis.

At the $3.8 \mu \mathrm{m}$-thick BOPP film side, the slope of the electric field from the internal interface to $11.8 \mu \mathrm{m}$ is almost the same as that in $25^{\circ} \mathrm{C}$ case. However, the positive slope region, which represents positive charge accumulation, extends from $3.8 \mu \mathrm{m}$ thick BOPP film side into more than middle depth of $9.8 \mu \mathrm{m}$ thick BOPP film. This proves again that higher temperature leads to more charge injection.

Charge mobility $\mu$ is the function of temperature. The function is as follows:

$$
\mu=\mu_{0} \frac{N}{M} \exp (-U / k T)
$$

where $\mu_{0}$ is the mobility ratio of free carriers that is a constant weakly dependent on temperature; $N, M, U$ and $k$ are the degeneracy (number of vacancies) of the conduction level, the number of traps, the level of traps, and the Boltzmann's constant, respectively. According to Equation (1), regardless of charge polarity, higher temperature leads to greater migration speed (for a given field value). This is believed the reason that both polarity injected charge extends to deeper distance as shown in Figure 5. Positive charges have greater mobility than negative ones [24].

Surprisingly, during the migration of positive charge at $40^{\circ} \mathrm{C}$, the internal interface does not show clear blocking effect. In general, the discontinuous region seems to be acting as a region with charge traps [10,11]. The continuous distribution of electric field may imply that the barrier at the interface can be neglected at this temperature and for this material. In other words, the blocking effect at the interface for space charge is very sensitive to the operating temperature.

\subsection{FIELD DISTRIBUTION IN SAMPLE A AT $70^{\circ} \mathrm{C}$}

Figure 6 shows the evolution of electric field distribution at $70^{\circ} \mathrm{C}$ within 2 hours under the applied electric field of 150 
$\mathrm{V} / \mu \mathrm{m}$. It is clear that serious distortion of the electric field has occurred in the sample A at $70^{\circ} \mathrm{C}$. As time goes on, the region with an oblique electric field gradually penetrates into the deeper distance on the $9.8 \mu \mathrm{m}$-thick BOPP film side. After sample A was subjected to applied field for two hours, the electric field with negative slope fills the whole layer. The mean accumulating negative charge density further enhances up to about $350 \mu \mathrm{C} / \mathrm{cm}^{3}$. Meanwhile, the electric field distribution curve with positive slope in the $3.8 \mu \mathrm{m}$-thick BOPP film bulk gradually tends to flatten, which implies the decreasing positive charge density from an initial value of about $180 \mu \mathrm{C} / \mathrm{cm}^{3}$. Even clearer asymmetrical charge injection with dominant negative charge is shown under this condition. The negative injected charge is detected in the whole $9.8 \mu \mathrm{m}$ layer but the region accumulating positive charge actually retracts at the elevated temperature. The confusing positive charge behavior may be explained as follows. Large amount of the injected positive charge migrates through the internal interface towards the cathode, as has already been observed at $40^{\circ} \mathrm{C}$. The enhancement of negative charge injection rate and mobility is expected in this case. Considering the asymmetric injection rate, negative charge is dominant and may even cross the internal interface. Positive and negative injected charges may recombine or spatially overlap in the deeper bulk. Therefore, a gradual reduction of the positive charge density from the 3.8 $\mu \mathrm{m}$ film side occurs as well as the narrowed positive charge region. In this case, the blocking effect of the internal interface for charge migration is completely gone.

Figure 7 shows the decaying electric field distribution at $70^{\circ} \mathrm{C}$ within 2 hours after the removal of applied voltage. The maximum of the residual electric field is about $80 \mathrm{~V} / \mu \mathrm{m}$. The slope of the residual electric field is almost the same by comparing the last curve under applied voltage and the first one under voltage-off, which means the same residual charge. Unlike the cases at 25 and $40^{\circ} \mathrm{C}$, the residual electric field curve at $70^{\circ} \mathrm{C}$ presents a distinct decaying trend particularly in the 9.8 $\mu \mathrm{m}$ film. As the thermal pulse measurement in this work is in one dimension, a plane parallel to film surface whose electric field equals zero is defined as zero electric field plane. In Figure 7 , the plane is at about $7 \mu \mathrm{m}$ (x- axis). After the removal of

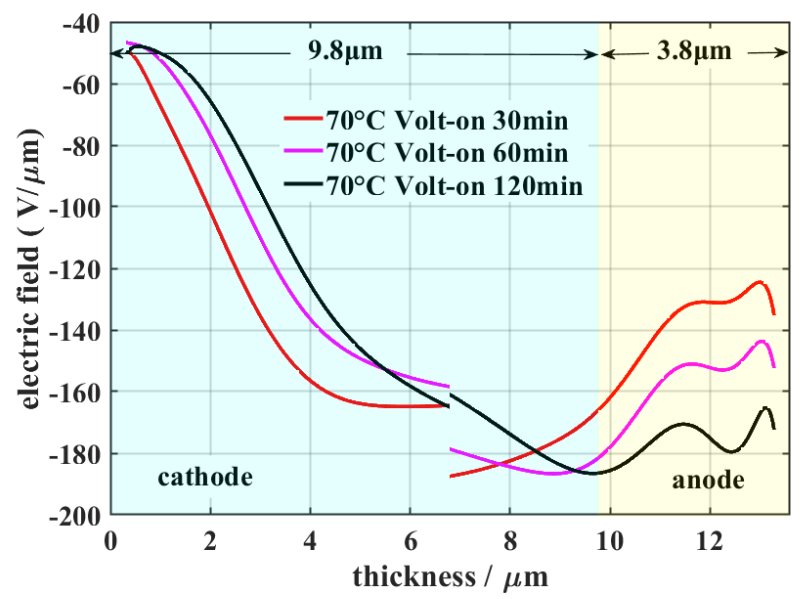

Figure 6. Electric field distribution of sample A during voltage-on at $70{ }^{\circ} \mathrm{C}$.

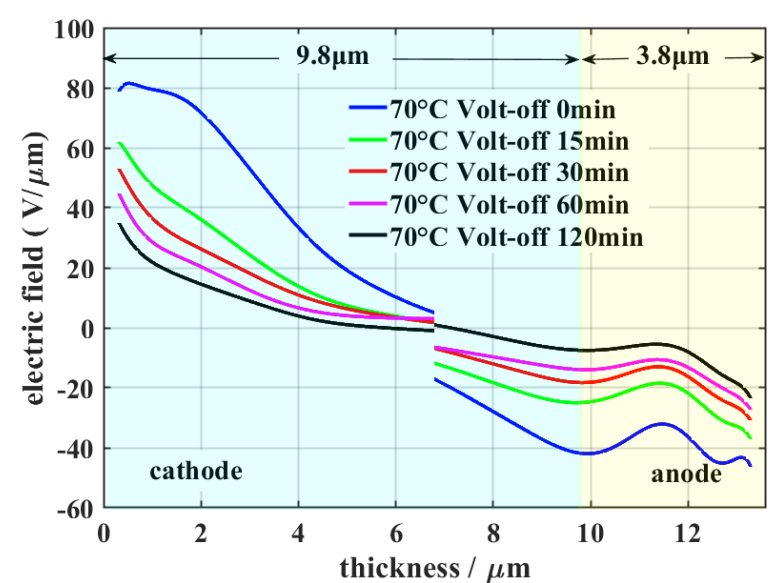

Figure 7. Electric field distribution of sample A during voltage-off at $70{ }^{\circ} \mathrm{C}$.

applied voltage, the negative charge in the left side of the zero electric field plane migrates towards the cathode, and the negative charge in the right side of the zero electric field plane migrates towards the original anode. The position of the zero electric field plane remains basically unchanged during the decay of the charge.

\section{DISCUSSION}

\subsection{SPACE CHARGE IN SAMPLE A}

Figure 8 shows the characterization results of the electric field within sample A at the moment of the removal of applied voltage at various temperatures. In 25 and $40{ }^{\circ} \mathrm{C}$ cases, $9.8 \mu \mathrm{m}$ film side mainly shows negative slope and positive slope, which means homopolar charge injection and accumulation. However, the elevated temperature at $70^{\circ} \mathrm{C}$ changes the status of doubleside homopolar charge accumulation. The bulk of the sample A is dominated by negative charge with very few positive charge in the middle part. Space charge injection and migration processes may lead to various electric field profiles when considered as function of the experimental temperature. Under the experimental conditions in this paper, the charge injection may be attributed to Schottky effect. The emission current density caused by the Schottky effect follows the RichardsonSchottky equation, which states that the charge emission intensity is enhanced by increasing the applied electric field intensity or elevating the experimental temperature [25]. The latter mechanism known as the thermal-aided Schottky effect reveals that positive and negative charge injection rate should simultaneously be enhanced at higher operating temperature. Furthermore, under given applied field intensity, the charge migration speed linearly related to the mobility should also increase as the temperature increases [24]. Therefore, one can expect that more space charge would be injected from the anode and the cathode to present as bipolar injection feature, and migration of charge should be faster with the temperature raising, which leads to different position of net charges and possibly to reduction of the accumulation of certain polar charges even under applied field. Besides the temperature, the blocking effect of the internal interface should be another 


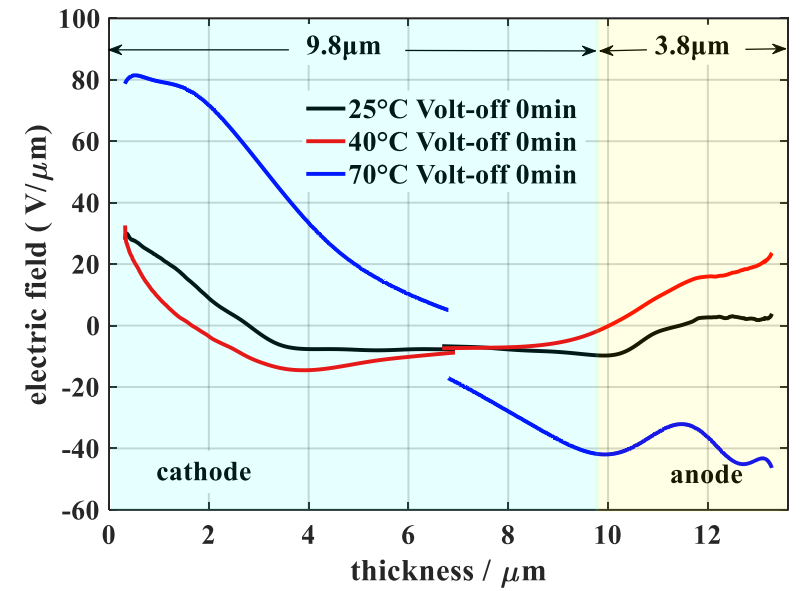

Figure 8. Electric field distribution of sample A immediately after the removc of the applied field $150 \mathrm{~V} / \mu \mathrm{m}$ at $25^{\circ} \mathrm{C}, 40^{\circ} \mathrm{C}$ and $70^{\circ} \mathrm{C}$, respectively.

significant factor to influence the space charge profiles. All of the factors jointly determine the space charge behavior.

Considering negative charges, the negative slope of the residual electric field near the surface of the $9.8 \mu$ m-thick BOPP film becomes sharper when the temperature rises from 25 to 40 ${ }^{\circ} \mathrm{C}$. This means that the accumulating negative charge increases according to the Poisson's equation, and further shows that the cathode injects more negative charges during voltage-on. At the same time, the migration depth of the negative charge is from $3.5 \mu \mathrm{m}$ depth at $25^{\circ} \mathrm{C}$ to $4 \mu \mathrm{m}$ depth at $40^{\circ} \mathrm{C}$, which indicates that slightly faster migrating speed has occurred. At $70^{\circ} \mathrm{C}$, the negative slope of the residual electric field near the surface of the $9.8 \mu \mathrm{m}$ BOPP film becomes smoother, but this does not mean lower injection rate with increasing temperature since the region with negative slope has extended to the internal interface. The negative slope can even been observed adjacent to the outer surface of $3.8 \mu \mathrm{m}$ film. The negative charge is thought to move from the cathode. Both of the phenomena at $70{ }^{\circ} \mathrm{C}$ suggest the enhanced injecting rate and higher mobility. The higher residual field at the cathode, which reflects the image of internal charges is a further prove of a higher amount of charges at $70^{\circ} \mathrm{C}$. But it is still not clear how far the internal interface influences negative space charge behavior.

From the $3.8 \mu \mathrm{m}$ film side, the region with positive slope of the residual electric fields extends to cross the internal interface at $40^{\circ} \mathrm{C}$. In contrast to this case, the positive slope stops at the interface both in the $25^{\circ} \mathrm{C}$ and $70^{\circ} \mathrm{C}$ cases, which cannot all be attributed to the blocking effect of the interface.

As discussed above, the effect of the experimental temperature on space charge behavior is clear. The role of the internal interface on space charge behavior can be explained as follows. From Figures 4-7, one can deduce that the electric field intensity at the internal interfaces is never less than the mean field applied during polarization at the various temperatures. Considering the interleaved electrode structure, there are sufficient reasons to believe that charges that can be injected into the dielectric medium from the external electrode can also migrate from the inside of the dielectric medium to the metal electrode within the internal interface. From this perspective, the internal interface should have no greater blocking effect on the transfer of charge than that at the outer interfaces. However, one should keep in mind that there is a very thin dielectric layer of DBT oil within the internal interface. The charge moving across the interface between the DBT oil and the evaporated aluminum must overcome the interface barrier. According to the above data, we may deduce that the interface barrier is too high for positive charge to overcome at $25^{\circ} \mathrm{C}$. As the temperature rises to $40^{\circ} \mathrm{C}$, the thermal-aided emission would get much enhanced to help the charge cross the oil-metal interface. That's believed to be the reason for the presence of positive charge adjacent to the internal interface within the $9.8 \mu \mathrm{m}$ film side. From another perspective, we may conclude that the barrier height of the oil-metal interface should be slightly higher than that of the thin BOPP film and metal interface. More attention should be paid to the coupling material when one considers space charge behavior at the interface. As for the experimental temperature up to $70^{\circ} \mathrm{C}$, the thermal-aided emission will of course be further enhanced, and both of the injected negative charge and positive can easily cross the oil-metal interface. The blocking effect of the interface barrier could be almost neglected under this kind of condition. Considering the asymmetrical injecting rate, large amount of negative charge could migrate to the vicinity of the anode and recombine or spatially overlap with positive charge. That's why we finally find the negative charge close to the anode.

\subsection{COMPARISON BETWEEN DIFFERENT INTERFACE STRUCTURES}

In order to further clarify the role of the internal interface on space charge behavior, the bilayer sample B thermally coupled by DBT thin layer without the internal metal electrodes is studied. The sample B is initially subjected to the applied field of $150 \mathrm{~V} / \mu \mathrm{m}$ for 2 hours at $25^{\circ} \mathrm{C}$, and then the residual field within the sample is monitored for another 2 hours. The electric field profiles are shown in Figure 9. From the $9.8 \mu \mathrm{m}$ side, there is clear negative slope region from the surface to about $3.5 \mu \mathrm{m}$ depth. This is similar to the sample A case at $25^{\circ} \mathrm{C}$. However, unlike the case in the sample A, the positive slope in the sample B doesn't stop at the internal interface, but extends to the 9.8 $\mu \mathrm{m}$ layer. This behavior confirms the deduction that the internal

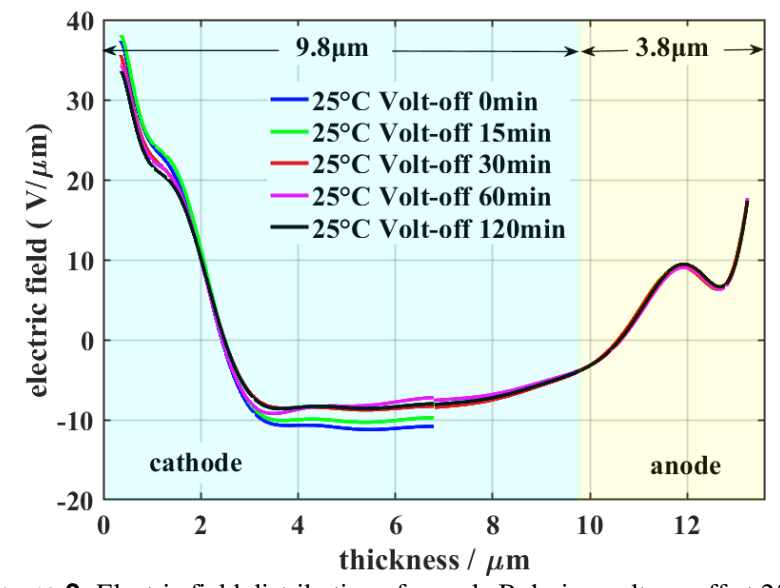

Figure 9. Electric field distribution of sample B during voltage-off at $25^{\circ} \mathrm{C}$. 
metal interface in the sample A blocks partially the positive charge.

What makes the difference in results of sample A from that of sample B must be attributed to the internal interface. We have observed the asymmetrical space charge build-up in the samples, which is dominated by negative charge. In other words, it means the metal-BOPP interface has partial blocking effect for both polarities charges and the barrier for positive charges is higher than that for negative ones. Therefore, according to the energy band theory, the energy level diagrams for a contact between Al and BOPP is as shown in Figure 10a and 10b. For positive charges (holes) injected from Al to BOPP by overcoming the barrier, they can easily shift from BOPP to Al. Considering the blocking phenomenon found in Sample A, the Al-DBT interface is therefore thought to be at the origin of the blocking effect. As discussed above, the electric field at the internal interface is not less than the mean field being subjected during polarization, therefore we believe that the barrier between Al-DBT interface should be higher than that between Al-BOPP, otherwise positive charge can go through the AlDBT interface at $25^{\circ} \mathrm{C}$. The positive charge goes through AlDBT interface and Al-BOPP interface should overcome the barriers:

$$
\begin{gathered}
\Delta E_{F V A l-D B T}=E_{F A l}-E_{V D B T} \\
\Delta E_{F V A l-B O P P}=E_{F A l}-E_{V B O P P}
\end{gathered}
$$

So,

$$
\triangle E_{F V A l-D B T}>\Delta E_{F V A l-B O P P}
$$

$\triangle E_{F V A l-D B T}$ and $\triangle E_{F V A l-B O P P}$ are the barrier height for positive charge to move through the Al-DBT interface and the Al-BOPP interface, respectively. Supposing DBT and BOPP have the same electron affinity $\chi$, we therefore deduce that the Fermi level of DBT should be lower than that of BOPP. According to energy band theory, the Fermi levels in two contacting material would be aligned to the same height. Figures 10c and 10d show the energy level diagrams for the contact between BOPP and DBT, and the barrier height:

$$
\triangle E_{V B O P P-V D B T}=E_{V B O P P}-E_{V D B T}
$$

should be overcome when the positive charge goes through the interface from BOPP to DBT. When Equation (2) subtracts Equation (5), we obtain:

$$
\Delta E_{F V A l-D B T}-\Delta E_{V B O P P-V D B T}=E_{F A l}-E_{V B O P P}>0
$$

It is clear that the barrier height for positive charge to overcome the Al-DBT interface is higher than that for BOPPDBT interface. It is thought to be the reason that positive is blocked at the Al-DBT interface in sample $\mathrm{A}$ at $25^{\circ} \mathrm{C}$ and extends through the BOPP-DBT interface in sample B. Therefore, the coupling medium between interfaces can be a critical factor for space charge behavior.

\section{CONCLUSIONS}

In this paper, the effect of the experimental temperature and the properties of internal interfaces on space charge characterization in bilayer BOPP films are investigated by improved thermal pulse method. The asymmetrical bi-polar space charge injection with dominant negative charge is found

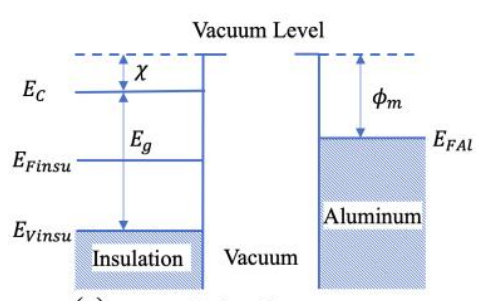

(a)

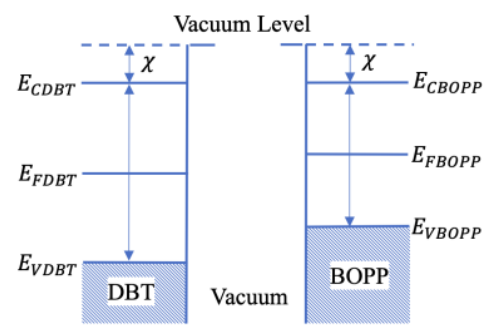

(c)

Before Contact

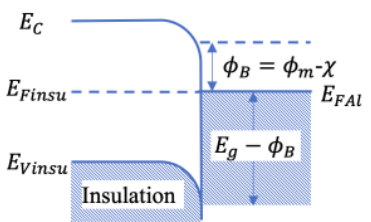

(b) After Contact

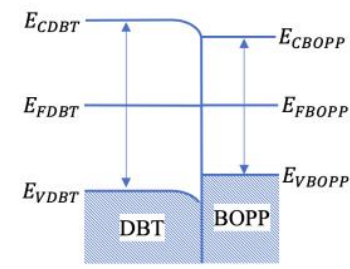

(d)
Figure 10. Energy level diagrams for a blocking contact for positive charge (hole) from right side to left side (a) dielectric-metal before contact (b) dielectric-metal after contact (c) DBT-BOPP before contact (d) DBT-BOPP after contact.

in the bilayer samples in all the temperature cases from $25^{\circ} \mathrm{C}$ to $70^{\circ} \mathrm{C}$. Space charge injection rate and migration are greatly enhanced by elevating temperature. The blocking effect of the interfaces between two film sheets for migrating space charge shows high sensitive to the experimental temperature. Increasing temperature could make the blocking effect weaken or even disappear. This phenomenon has been observed when the temperature goes from $25^{\circ} \mathrm{C}$ up to $40^{\circ} \mathrm{C}$. The weakening of the blocking effect at higher temperature is attributed to the enhancement of thermal emission. Another factor to influence the blocking effect is the properties of the internal interfaces. The internal interface region actually includes several interfaces, and the difference of the energy level between two adjacent materials acting as barrier for the incoming charge is the critical parameter to decide the charge migration. The study indicates that the BOPP-DBT interface is easier for positive charge (hole) to go through than the Al-DBT interface.

\section{ACKNOWLEDGMENT}

One of the authors ( $\mathrm{F}$. Zheng) really appreciates the financial support from the National Natural Science Foundation of China (NSFC No. 51477119).

G. Teyssedre is grateful to Tongji University for the position of invited researcher awarded in the course of this work.

\section{REFERENCES}

[1] Z. Gong et al, "R\&D of composite thin films," Vacuum, vol. 43, pp. 19$22,2006$.

[2] M. A. Wolak et al, "Dielectric response of structured multilayered polymer films fabricated by forced assembly," Appl. Phys. Lett., vol. 92, p. 113301, 2008.

[3] L. Yu, "Thin-film capacitor and flat mini thin-film capacitor overview," Electronic Product World, Vol. 11,pp. 18-19, 2003.

[4] R. Bodega, " Space charge accumulation in polymeric high voltage DC cable system," $\mathrm{PhD}$ dissertation, The Netherlands: Delft University of Technology, 2006. 
[5] F. Rogti, A. Mekhaldi, C. Laurent, "Space Charge Behavior at Physical Interfaces in Cross-linked Polyethylene under DC Field," IEEE Trans. Electr. Insul.,vol. 15, pp. 1478-1485, 2008.

[6] R. Bodega et al, "Dielectric interface characterization by means of space charge measurements," Аппи. Rep. Conf. Electr. Insul. Dielect. Phenom. (CEIDP), 2003, vol. 1, pp. 728-733.

[7] M. Clausse, "Theoretical conditions for existence of a dielectricrelaxation due to maxwell-wagner effect in heterogeneous binarysystems and especially in emulsions," Colloid and Polym. Sci., vol. 253, pp. 1020-1024, 1975.

[8] J. H. Daly et al, "The dielectric-properties of polycarbonate styrene acrylonitrile copolymer multilayer composites .2. the maxwell-wagnersillars process," Journal of Materials Science Letters, vol. 11, pp. 12711273, 1992.

[9] R. Karthik, V. Tummala, "Voltage dependent maxwell-wagner polarization in dielectric heterostructures," Mater. Today-Proceedings, vol. 4, pp. 8751-8757, 2017.

[10] G. C. Montanari, P. H. F. Morshuis, "Space charge phenomenology in polymeric insulating materials," IEEE Trans. Electr. Insul., vol. 12, pp. 754-767, 2005.

[11] K. Wu, C. Cheng, "Interface Charges between Insulating Materials," IEEE Trans. Electr. Insul., vol. 24, pp. 2633-2642, 2017.

[12] T. Tanaka et al, "Characteristics of space charge formed in a laminated LDPE/EVA dielectric under DC stress," the IEEE Intl Symp. Electr. Insul.(ISEI), 1996, vol. 1, pp. 184-187.

[13] R. Bodega et al, "Charging and polarization phenomena in XLPE-EPR coaxial interfaces," Intl Symp. Electr. Insul. Mater. (ISEIM), 2005 vol. 1, pp. 107-110.

[14] L. Lan et al, "Space Charge Property at the Interface in Low Density Polyethylene/Ethylene Propylene Rubber Double-layered Insulation," Proceedings of the Chinese Society of Electrical Engineering, vol. 35, pp. 1266-1272, 2015.

[15] T. T. N. Vu et al, "Correlating Conductivity and Space Charge Measurements in Multi-dielectrics Under Various Electrical and Thermal Stresses," IEEE Trans. Electr. Insul., vol. 22, pp. 117-127, 2015.
[16] T. T. N. Vu et al, "Maxwell-Wagner Effect in Multi-Layered Dielectrics: Interfacial Charge Measurement and Modelling," Technologies, vol. 5, p. 5-27, 2017.

[17] S. Das, N. Gupta, "Interfacial Charge Behaviour at Dielectric Dielectric Interfaces," IEEE Trans. Electr. Insul., vol. 21, pp. 1302-1311, 2014.

[18] F. Zheng et al, "Thermal pulse measurements of space charge distributions under an applied electric field in thin films," Measurement Science and Technology, vol. 24, p. 065603, 2013.

[19] B. Ploss, R. Emmerich, S. Bauer, "Thermal wave probing of pyroelectric distributions in the surface region of ferroelectric materials - a new method for the analysis," J. Appl. Phys., vol. 72, pp. 5363-5370, 1992.

[20] M. Axel, "Unbiased iterative reconstruction of polarization and spacecharge profiles from thermal-wave experiments," Meas. Sci. Technol., vol. 15, pp. 1347-1353, 2004.

[21] A. Petre et al, "A Comparison of Different Mathematical Treatments for Solving the Inverse Problem in Focused Laser Intensity Modulation Method," Jpn. J. Appl. Phys., vol. 43, pp. 2572-2579, 2004.

[22] M. Liang, F. Zheng, Z. An, Y. Zhang, "Numerical extraction of electric field distribution from thermal pulse method based on Monte Carlo simulation," Acta Physica Sinica, vol. 65, p. 077702, 2016.

[23] K. C. Kao, Dielectric Phenomena in Solids, Elsevier Academic Press, 2004.

[24] F. Zheng et al, "Space Charge Characterization in Biaxially Oriented Polypropylene Films," IEEE Trans. Electr. Insul., vol. 23, pp. 31023107, 2016.

[25] P. Notingher et al, "Study of space charge accumulation in polyolefins submitted to ac stress," IEEE Trans. Electr. Insul., vol. 8, pp. 972-984, 2001. 